\title{
Scattering and depolarization of microwaves by spheroidal raindrops
}

\author{
C. Warner
}

Department of Meteorology, McGill University, Montreal, Quebec, Canada

\section{A. Hizal}

\author{
Department of Electrical Engineering, The Middle East Technical University, Ankara, Turkey
}

(Received May 17, 1976.)

\begin{abstract}
With a view to calculating depolarization and differential attenuation of microwaves by rain, with allowance for the variable shape and orientation of raindrops, the theory of $P$. $C$. Waterman is applied to the calculation of cross sections and depolarization ratios of spheroids, and drops of the shapes determined by H. R. Pruppacher and R. L. Pitter. Use of Waterman's extended boundary condition facilitates computation. The scattering characteristics of spheroids appear similar in nature to those of spheres, in the regimes considered. Marked singularities in the characteristics of backscattering depolarization appear at optical size 1.5. The Pruppacher and Pitter drops scatter similarly to equivalent oblate spheroids. Implications for weather radar measurements are briefly discussed.
\end{abstract}

\section{INTRODUCTION}

Attenuation and depolarization of microwaves by rain are being studied, to provide data for the design of terrestrial and satellite communication links, and as a basis for attempts to evolve radars capable of distinguishing between, and measuring accurately, different types of precipitation. The purpose of this paper is to establish a new base from which to calculate depolarization and differential attenuation, in rain with drops of spheroidal shapes and arbitrary orientations.

The theory of scattering by spheroidal raindrops has been developed by Oguchi [1973] and Oguchi and Hosoya [1974]. They have used a point-matching technique in which electric and magnetic fields are expanded in spherical vector wave functions. The coefficients of the latter two expansions are obtained from boundary conditions for representative points on the surface of the scatterer: no discontinuities of tangential components of the total fields occur across the surface. This point-matching technique has been refined by Morrison and Cross [1974], and several authors have observed attenuation and cross polarization of microwaves in rain [Chu, 1974; Taur, 1975]. By modelling the rain in terms of oblate spheroids, canted in a plane

Copyright (C) 1976 by the American Geophysical Union. containing the direction of propagation, or normal to it, some success has been achieved in comparing theory with observations. However, raindrops take on a variety of shapes [Jones, 1959], and the regimentation of raindrops according to a canting angle seems unsatisfactory.

In order to calculate cross polarization and attenuation using more realistic models of rainfall, involving drops with a variety of shapes and orientations, the theory of Waterman [1965, 1969] is applied to backscattering and extinction by spheroidal drops both oblate and prolate, and to the drops of the equilibrium shapes of Pruppacher and Pitter [1971]. The latter are similar to oblate spheroids, but with concavity on the underside. Waterman's theory appears easier to implement than the pointmatching technique, by virtue of the 'extended boundary condition' [ Waterman, 1965]. This method invokes equivalent electric and magnetic currents on the surface of the scatterer, producing fields which, according to the field equivalence principle, precisely cancel the incident fields throughout the interior volume; the boundary condition is referred to a spherical surface inscribed within the scatterer. After outlining the theory, and comparing results with other work, backscattering and extinction cross sections and depolarization ratios are calculated for a variety of drops, for incident polarization both linear and circular. 


\section{SCATTERING THEORY}

Scattering theory originally due to Waterman $[1965,1969]$ is outlined below. The time dependence is assumed to be of the form $\exp (-i k c t)$, where $k$ and $c$ respectively are the wave number and velocity of light in vacuo. This factor is omitted in equations for field quantities below. Spherical wave expansions correspond with those used by Hizal and Marincic [1970], except that here all spatial variables are normalized with $k$.

A scatterer of symmetry about an axis of rotation, and of uniform dielectric constant $\epsilon$, is considered. Its surface $(S)$ is assumed to be sufficiently regular that the divergence theorem is applicable, and a continuous, single-valued normal $\mathbf{u}_{n}$ is defined at each point. Spherical coordinates $(r, \theta, \phi)$ are used, with the origin inside the scatterer and the direction $\theta=0$ along the axis of symmetry (Figure 1).

The incident electric and magnetic fields may be expressed by the following expansions, representing solutions of the vector wave equation in spherical coordinates:

$$
\begin{aligned}
& \mathbf{E}^{i}=i Z_{0} \sum_{n=1}^{x} \sum_{m=-n}^{n} \sum_{p=1}^{2} y_{n m}^{i p} \mathbf{F}_{n m}^{1 q}(x, \theta, \phi) \\
& \mathbf{H}^{\prime}=\sum_{n=1}^{\infty} \sum_{m=-n}^{n} \sum_{p=1}^{2} y_{n m}^{i 1 p} \mathbf{F}_{n m}^{1 p}(x, \boldsymbol{\theta}, \phi)
\end{aligned}
$$

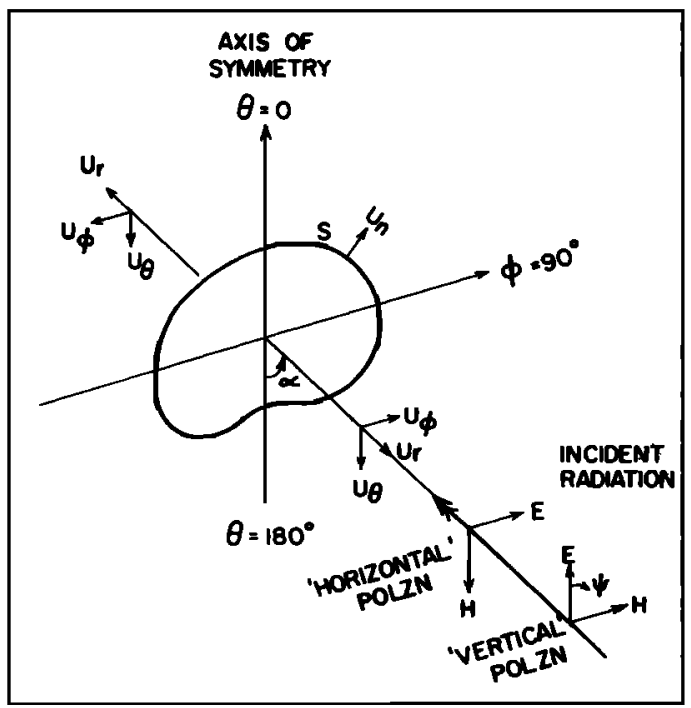

Fig. 1. Scattering geometry.
Here the constant $Z_{0}=\left(\mu_{0} / \epsilon_{0}\right)^{0.5}$ refers to free space. The superscript $p=1$ or 2 and

$$
q=3-p
$$

The coefficients $y_{n m}^{i 1 p}$ for $p=1$ and 2 correspond to electric and magnetic multipoles; the subscripts $m$ and $n$ refer to harmonics of azimuth and elevation respectively. $F$ are the spherical wave functions

$$
\begin{gathered}
\mathbf{F}_{n m}^{f 1}=-C_{n}^{m} z_{n}^{f} \mathrm{e}^{i m \phi}\left(\frac{m P_{n}^{m}}{\sin \theta} \mathbf{u}_{\theta}+i \frac{\mathrm{d} P_{n}^{m}}{\mathrm{~d} \theta} \mathbf{u}_{\phi}\right) \\
\mathbf{F}_{n m !}^{f 2}=C_{n}^{m} \mathrm{e}^{i m \phi}\left\{i n(n+1)\left(z_{n}^{f} / x\right) P_{n}^{m} \mathbf{u}_{r}+\left[\left(x z_{n}^{f}\right)^{\prime} / x\right]\right. \\
\left.\cdot\left[\left(i \mathrm{~d} P_{n}^{m} / \mathrm{d} \theta\right) \mathbf{u}_{\theta}-\left(m P_{n}^{m} / \sin \theta\right) \mathbf{u}_{\phi}\right]\right\}
\end{gathered}
$$

where $u$ are the unit vectors of spherical coordinates; $P_{n}^{m}(\cos \theta)$ is the associated Legendre function, and

$C_{n}^{m}=\left\{\frac{(2 n+1)}{4 \pi n(n+1)} \frac{(n-m) !}{(n+m) !}\right\}^{0.5}$

With $x=k r, z_{n}^{f}(x)$ is the spherical Bessel function for $f=1$, the spherical Hankel function of the first kind for $f=s$, and of the second kind for $f=2$. The prime in (3) denotes differentiation with respect to $x$. The use of $f=1$ only in (1) means restriction to incoming waves, finite at the origin within the scatterer, as opposed to a summation over both incoming and outgoing types.

The total electric and magnetic fields inside the scatterer are expressed as spherical waves:

$\mathbf{E}^{t}=i Z \sum_{n=1}^{\infty} \sum_{m=-n}^{n} \sum_{p=1}^{2} y_{n m}^{i t p} F_{n m}^{1 q}\left(x_{d}, \theta, \phi\right)$

with a similar equation for $\mathbf{H}^{\mathbf{t}}$. In this case $Z=$ $Z_{0} \epsilon^{-0.5}$, referring to the scatterer, and $x_{d}=\epsilon^{0.5}$ $\mathrm{kr}$, replacing $x$ as the argument of the Bessel functions.

Finally, scattered fields in vacuo are expressed as spherical waves:

$\mathbf{E}^{a}=i Z_{0} \sum_{n=1}^{\infty} \sum_{n=-n}^{n} \sum_{p=1}^{2} y_{n m}^{a p} \mathbf{F}_{n m}^{a q}(x, \theta, \phi)$

with a similar equation for $\mathbf{H}^{a}$. Outside the largest sphere enclosing the scatterer, the waves are outgoing at infinity so as to satisfy the radiation condition, and multipole coefficients $y_{n m}^{s p}$ are used. For the 
scattered waves which are incoming, the multipole coefficients are $y_{n m}^{1 p}$.

Now the conditions on the boundary $S$ are considered, or rather, on a surface $S^{\prime}$ displaced outward from $S$ across a small vacuum gap. Equivalent electric and magnetic sources exist on $S^{\prime}$, related to the total fields as follows:

$$
\begin{gathered}
\mathbf{K}^{E}=\mathbf{u}_{n} \times \mathbf{H}^{t} \\
\mathbf{K}^{M}=-\mathbf{u}_{n} \times \mathbf{E}^{\prime}
\end{gathered}
$$

Outside $S^{\prime}$, these sources yield the scattered fields. Inside $S^{\prime}$ they precisely cancel the incident fields. This is Waterman's [1965] extended boundary condition.

Tangential components of $\mathbf{H}^{t}$ and $\mathbf{E}^{t}$ are continuous across $S$, so that the scattered fields both interior and exterior to $\dot{S}$ may be obtained from the sources $\mathbf{K}$, through the free space dyadic Green's function. Expanding the latter in spherical waves [Morse and Feshbach, 1953] it is found that

$y_{n m}^{a p}=\int_{S}\left(i K^{E} \cdot F_{n m}^{b q^{*}}-Z_{0}^{-1} K^{M} \cdot F_{n m}^{b p^{*}}\right) d s_{k}$

where $\mathrm{d} s_{k}=k^{2} \mathrm{~d} s$ is the normalized surface element, and the asterisk means complex conjugation (not involving the argument $x_{d}$ of the radial functions, which may be complex if the dielectric is lossy).

$$
b=2-\delta_{a s}
$$

where $\delta_{a s}$ is Kronecker's delta, and $q$ is given by (2).

From the extended boundary condition, the scattered fields within $S$ precisely cancel the incident fields. We refer this to a spherical surface within $S$, centered at the origin. The orthogonality properties of the vector wave functions over a spherical surface then allow one to write

$$
y_{n m}^{1 p}=-y_{n m}^{i p p}
$$

By analytic continuation arguments, it follows that this relationship is valid everywhere within $S[\mathrm{Wa}$ terman, 1965].

After substituting (5) and (7) in (8), the following matrix equations are obtained for the coefficients of the scattered fields respectively inside and outside $S$ :

$$
W_{n n^{\prime} n m^{\prime}}^{11 p p^{\prime}}, y_{n^{\prime} m^{\prime}}^{11 p^{\prime}}=y_{n m}^{1 p}=-y_{n m}^{i 1 p}
$$

$W_{m r^{\prime} m m^{\prime}}^{s l p p^{\prime}}, y_{n^{\prime} m m^{\prime}}^{t l p^{\prime}}=y_{n m}^{s p}$

where $p^{\prime}=1,2 ; n^{\prime}=1,2, \ldots$, and $m^{\prime}=-n^{\prime}$, $\ldots, 0, \ldots, n^{\prime}$ are the independent indices over which summation is carried out. For rotational bodies, which are considered here, $m=m^{\prime}$. The coefficients for $m<0$ are related to those for $m>0$ by the property

$C_{n}^{-m} P_{n}^{-m}=(-1)^{m} C_{n}^{m} P_{n}^{m}$

This reduces the number of equations to be solved. Further, since the Legendre functions $P_{n}^{m}$ are zero for $m>n$, then for each azimuth mode $m>1$, elevation modes $n$ are started from the value of $m$, rather than from 1 . Only sufficient terms are evaluated to arrive asymptotically at settled results, as indicated in appendix A. Where $m_{1}$ is the chosen number of azimuth modes (the first corresponding to $m=0$ ), and $n_{1}$ is the chosen number of elevation modes (starting from $m$ ), the number of equations in each of (11) and (12) is $2 m_{1} n_{1}$. The scattering matrices are given by

$$
\begin{aligned}
& W_{m r^{\prime} m m^{\prime}}^{a l l p^{\prime}}=-i \int_{S}\left\{F_{m m}^{b q^{*}} \times F_{n^{\prime} m^{\prime}}^{\mid p^{\prime}}\left(x_{d}\right)\right. \\
& \left.+\epsilon^{-0.5} \mathbf{F}_{n m}^{b p^{*}} \times \mathbf{F}_{n^{\prime} m^{\prime}}^{l q^{*}}\left(x_{d}\right)\right\} \cdot \mathbf{u}_{n} d s_{k}
\end{aligned}
$$

where the pairs $q, p$ and $q^{\prime}, p^{\prime}$ independently satisfy (2), and $b$ is given by (9). The coefficients $y_{n^{\prime} m^{\prime}}^{t p^{\prime}}$ are found from (11), and then the coefficients $y_{n m}^{s p}$ of the scattered fields from (12). This completes the formulation of the problem.

\section{CROSS SECTIONS AND DEPOLARIZATION RATIOS}

For individual particles of prescribed size and shape, for a particular frequency, cases are considered of linearly polarized plane waves incident at angle $\alpha$ (Figure 1). The $E$ field is normal to the axis of the scatterer, with 'vertical $(V)$ ' polarization, or it has a 'horizontal $(H)$ ' direction. In the former case, the multipole coefficients of the incident radiation are given by

$y_{n m}^{i l l}=4 \pi C_{n}^{m} i^{(2 m+n-1)}\left(d P_{n}^{m} / d \theta\right)_{\theta=\alpha}$

$y_{m m}^{i 12}=4 \pi C_{n}^{m} i^{(2 m+n-1)} m P_{n}^{m}(\cos \alpha) / \sin \alpha$

In the latter case, the right hand sides of these equations are interchanged. Outgoing scattered farfields as follows are found for these two polarizations: 


$$
\begin{aligned}
\mathbf{E}_{v}^{s} & =Z_{0} \frac{\mathrm{e}^{i x}}{x} \sum_{n=1}^{\infty} \sum_{m=0}^{\mathrm{n}}\left(2-\delta_{m \theta}\right)(-i)^{n+1} C_{n}^{m}\left[\left(y_{n m}^{s 2} m P_{n}^{m}\right.\right. \\
& \left.\div \sin \theta+y_{n m}^{s 1} \mathrm{~d} P_{n}^{m} / \mathrm{d} \theta\right) \cos m \phi \mathbf{u}_{\theta}-\left(y_{n m}^{s 2} \mathrm{~d} P_{n}^{m}\right. \\
& \left.\left.\div \mathrm{d} \theta+y_{n m}^{s 1} m P_{n}^{m} / \sin \theta\right) \sin m \phi u_{\phi}\right]=Z_{0}\left(\mathrm{e}^{i x} / x\right) \\
& {\left[\left(A_{v_{\theta 0}}+i A_{V \theta 1}\right) \mathbf{u}_{\theta}+\left(A_{V \phi 0}+i A_{V \phi 1}\right) \mathbf{u}_{\phi}\right] } \\
\mathbf{E}_{H} & =Z_{0} \frac{\mathrm{e}^{i x}}{x} \sum_{n=1}^{\infty} \sum_{m=0}^{n}\left(2-\delta_{m \theta}\right)(-i)^{n+1} C_{n}^{m}\left[\left(y_{n m}^{s 2} m P_{n}^{m}\right.\right. \\
& \left.\div \sin \theta+y_{n m}^{s 1} \mathrm{~d} P_{n}^{m} / \mathrm{d} \theta\right) \sin m \phi \mathbf{u}_{\theta}+\left(y_{n m}^{s 2} \mathrm{~d} P_{n}^{m} / \mathrm{d} \theta\right. \\
& \left.\left.+y_{n m}^{s 1} m P_{n}^{m} / \sin \theta\right) \cos m \phi \mathbf{u}_{\phi}\right]=Z_{0}\left(\mathrm{e}^{i x} / x\right)\left[\left(A_{H \theta 0}\right.\right. \\
& \left.\left.+i A_{H \theta 1}\right) \mathbf{u}_{\theta}+\left(A_{H \phi 0}+i A_{H \phi 1}\right) \mathbf{u}_{\phi}\right]
\end{aligned}
$$

In these equations $x$ is the optical distance from the origin of coordinates within the scatterer; here $x$ is large. The factor $\left(2-\delta_{m_{0}}\right)$ caters for the cases of $m<0$. The components $A$ of amplitude are identified, to find depolarization.

Backscattering $(B)$ and extinction $(E)$ cross sections, normalized with respect to the equivolume spherical diameter $(d)$ of the scatterer, for each polarization, are obtained from the expressions

$\sigma_{B \alpha V}=\frac{16}{k^{2} d^{2}}\left|Z_{0}^{-1} x \mathrm{e}^{-i x} \mathbf{E}_{V}^{s}(\pi-\alpha, 0)\right|^{2}$

$\sigma_{E \alpha V}=\frac{16}{k^{2} d^{2}} \operatorname{Re}\left(Z_{0}^{-1} x \mathrm{e}^{-i x} \mathbf{E}_{V}^{s}(\alpha, \pi) \cdot u_{\theta}\right)$

$\sigma_{E \alpha H}=\frac{16}{k^{2} d^{2}} \operatorname{Re}\left(Z_{0}^{-1} x \mathrm{e}^{-i x} \mathbf{E}_{H}^{s}(\alpha, \pi) \cdot u_{\phi}\right)$

where $E_{v}^{s}(\theta, \phi)$ is the scattered field along the direction of elevation $\theta$ and azimuth $\phi$, and $\alpha$, the angle of incidence, is varied in the computations. The expression for $\sigma_{B \alpha H}$ is similar to that for $\sigma_{B \alpha V}$.

If only backscattering and extinction are considered, the amplitudes

$A_{V \phi O}=A_{V_{\phi 1}}=A_{H \theta 0}=A_{H \theta 1}=0$

For the general case of linear polarization not along the direction $V$ or $H$, but at an angle $\psi$ from the $V$ direction, it is found that

$\sigma_{B \alpha \psi}=\cos ^{2} \psi \sigma_{B \alpha V}+\sin ^{2} \psi \sigma_{B \alpha H}$

and similarly for $\sigma_{E \alpha \psi}$.

The depolarization ratio $(D R)$ is defined as the ratio of the power in the orthogonal component to that in the main component of the scattered radiation. Then for back $(B)$ or forward $(F)$ scatter- ing with linear $(L)$ polarization, of orientation $\psi$, the two depolarization ratios

$$
\begin{aligned}
& B L D R \\
& F L D R
\end{aligned}=\frac{\left(A_{V \theta 0} \pm A_{H \phi 0}\right)^{2}+\left(A_{V \theta 1} \pm A_{H \phi 1}\right)^{2}}{\left(A_{V \theta 0} / t \mp A_{H \phi 0} t\right)^{2}+\left(A_{V \theta 1} / t \mp A_{H \phi 1} t\right)^{2}}
$$

the upper and lower signs referring to $B L D R, F L D R$ respectively, with $t=\tan \psi$. For circular $(C)$ polarization, cross sections, and circular depolarization ratios $B C D R$ and $F C D R$, are obtained by setting $\psi=45^{\circ}(t=1)$. At a certain value $\psi$ $=\Psi_{m}, L D R$ reaches a maximum:

$$
L D R_{m}=C D R(1+X)
$$

This maximum is given by

$\tan ^{4} \psi_{m}=\left(A_{v \theta 0}^{2}+A_{v \theta 1}^{2}\right) /\left(A_{H \phi 0}^{2}+A_{H \phi 1}^{2}\right)$

and

$X=\frac{0.5\left(1-\cot ^{2} \psi_{m}\right)^{2}}{\cot ^{2} \Psi_{m} \mp\left(\frac{A_{V \theta 0} A_{H \phi 0}+A_{V \theta 1} A_{H \phi 1}}{A_{V \theta 0}^{2}+A_{V \theta 1}^{2}}\right)}$

the upper and lower signs $\mp$ referring, respectively, to back and forward scattering.

Calculations were made for temperature $10^{\circ} \mathrm{C}$ using dielectric constants obtained from parameters given by Buckley and Maryott [1958], which are close to those of Ray [1972].

The computation proceeds with specification of the shape of the scatterer at 32 Gauss nodes [Abramowitz and Stegun, 1968], with numerical integration for the coefficients $y_{n^{\prime} m^{\prime}}^{t p^{\prime}}$ of equations (11). Double precision (64 bit) arithmetic is used, on an IBM computer. Bessel functions are calculated after the manner of Shafai et al. [1970]. An angle $\alpha$ of incidence is specified, equations (12) are solved, again using double precision arithmetic, and expressions (16) through (18) are evaluated, and (20), with $t=1$, through (23).

For obtaining cross sections of spheroidal water drops to a precision of three significant figures, required numbers $\left(m_{1}, n_{1}\right)$ of azimuth and elevation modes are given in appendix $\mathrm{A}$. While Morrison and Cross [1974] used values of $\left(m_{1}, n_{1}\right)$ up to $(9,23)$ at $30 \mathrm{GHz}$ with the point-matching technique, here it was found necessary to go only as far as $(7,14)$, so that Waterman's theory seems easier to use in these applications. 


\section{VERIFICATION OF THE THEORY}

Several tests of the scattering computation were performed. Results for spheres, with centers displaced from the origin, corresponded very precisely with those obtained from Mie theory for spheres, and the reciprocity theorem of bistatic scattering was found to be satisfied. Waterman [1969] treated a sphere-cone-sphere body of optical length 2.6, and 2:1 prolate spheroids, both with $\epsilon=4$. His curves for the former body (his Figure 4) were reproduced accurately, using $m_{1}=3$ and $n_{1}=$ 10. Computations for the largest prolate spheroid (his Figure 5), of optical length 5, showed agreement in general features, but a discrepancy ( 0.34 instead of 0.22 ) in $\sigma_{B}$ near 'aspect angle' $30^{\circ}$. (It was found necessary to take $m_{1}=6$ and $n_{1}=15$ to get $\sigma_{B}$ for aspect angle 0 accurate to \pm 15 percent.) Calculations of Oguchi [1973] were reproduced very closely, confirming that the accuracy of the point-matching technique is better than those of his alternative methods. Results of laboratory mea-

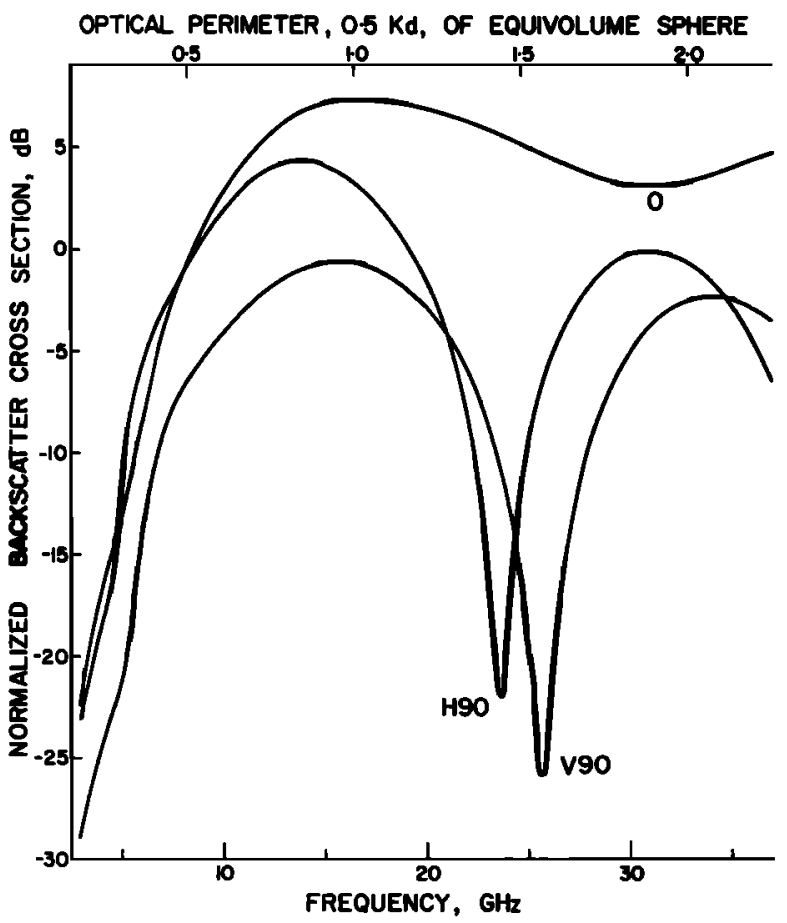

Fig. 2. Scattering cross sections for a drop of diameter $5.8 \mathrm{~mm}$, $b / a=0.55$, normalized by the cross section of an equivolume sphere; backscatter. Labels V90, H90: vertical, horizontal polarization, respectively, at incidence $\alpha=90^{\circ}$. Label 0: linear polarization with $\alpha=0$. surements of Atlas and Wexler [1963], of backscattering by oblate spheroids made of a material like ice, were reproduced to within the limits of accuracy of the measurements.

\section{RESULTS: SCATTERING BY RAINDROPS}

Jones [1959] has measured the shapes of raindrops, and Pruppacher and Pitter [1971] have photographed drops in a wind tunnel, and calculated equilibrium shapes, as a function of size, for drops falling steadily. After the work of Jones, spheroidal drops are treated, with axis/diameter ratios from 0.55 to 1.3 , nearly covering his observed range. Scattering by the drops of Pruppacher and Pitter also is included. In a companion paper [Warner, 1975] following upon these results, ways have been found of dealing simply with the dependence of cross section and depolarization ratio on drop shape and orientation.

A large oblate raindrop, of diameter $5.8 \mathrm{~mm}$ and axis / diameter $b / a=0.55$, is considered first. Normalized backscatter cross sections of it are plotted as a function of frequency in Figure 2; normalized extinction cross, sections are plotted in Figure 3 (which may be compared with results of

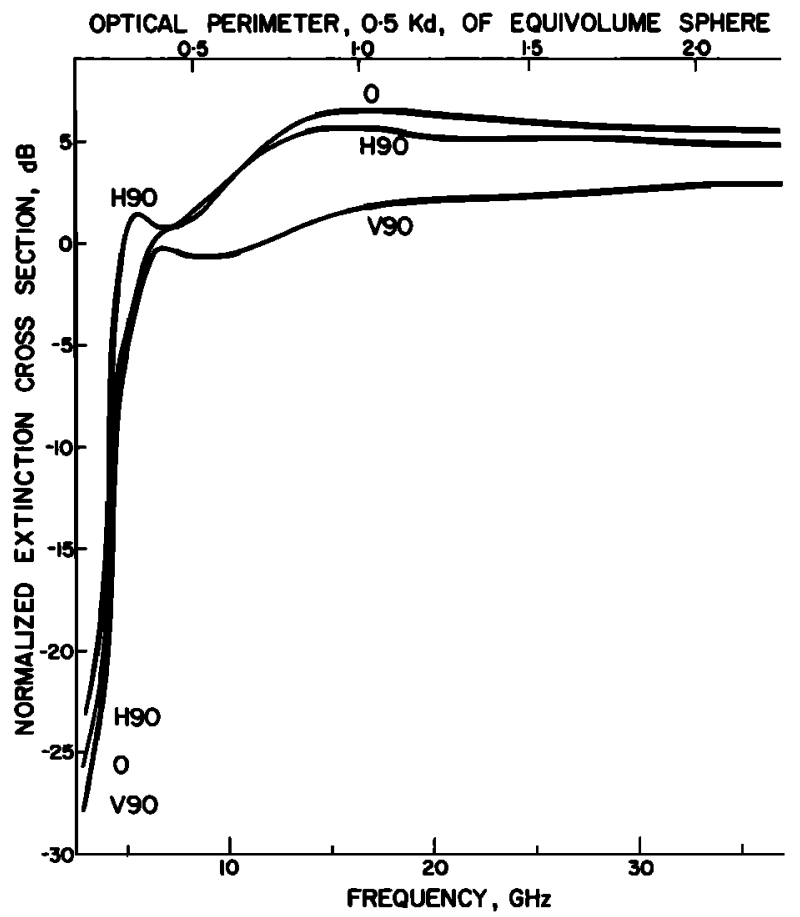

Fig. 3. See caption for Figure 2; extinction. 
Oguchi [1964]). The trios of curves $V 90, H 90$, and 0 refer respectively to incidence with vertical linear polarization $(\psi=0)$ at $\alpha=90^{\circ}$, horizontal polarization $\left(\psi=90^{\circ}\right)$ with $\alpha=90^{\circ}$, and linear polarization with $\alpha=0$ (looking along the axis of symmetry). The curves of Figures 2 and 3 resemble those of Mie scattering by spheres, but the minima in the curves of backscattering are very pronounced at $\alpha=90^{\circ}$, and not so at $\alpha=0$. The opposite is the case with prolate drops. There is no very simple explanation for the curves, but one notes that Inada and Plonus [1970] have explained Mie curves for certain dielectric spheres in terms of surface waves, which partially penetrate into the sphere and undergo internal reflection. The pronounced minima of backscattering shown in Figure 2 occur at optical perimeter $0.5 k d \simeq 1.5$, which suggests that their explanation may be applicable to these cases also.

Striking results are obtained for depolarization ratios. For two sizes of large oblate drop, $B C D R$ and $B L D R_{m}$ (equations (20) through (23)) are shown in Figure 4 as a function of frequency. At optical perimeter 1.5 , the rising of these two ratios above $0 \mathrm{~dB}$ is a result of decrease of the main component, rather than increase of the orthogonal component, of the scattered radiation. Both this effect, and at lower frequencies the lesser maxima and minima of the curves, are associated with certain of the components $A$ of the scattered fields going through zero. With $b / a$ closer to 1.0 the effects are less marked. Depolarization ratios for prolate drops

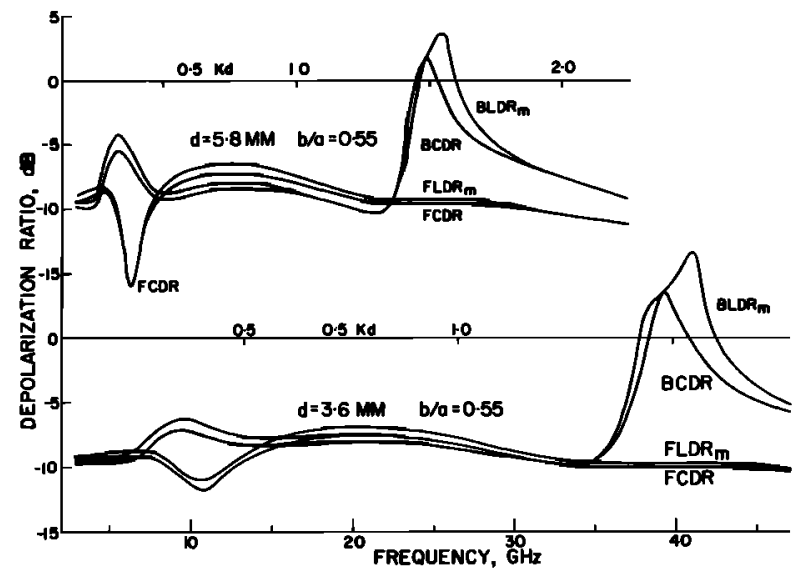

Fig. 4. Depolarization ratios $(D R)$ for drops of diameters 5.8 and $3.6 \mathrm{~mm}$, with $b / a=0.55$. $B, F$ : back, forward scattering, respectively. Labels $C, L$ : circular, linear polarization, respectively. Values of $B L D R$ are maxima.

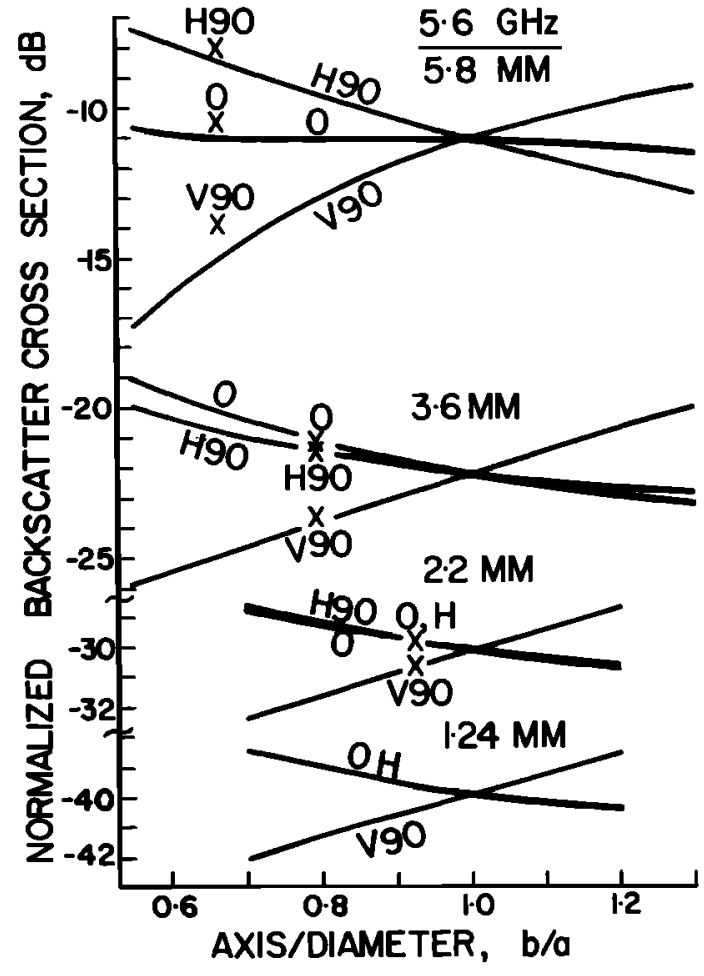

Fig. 5. Normalized cross sections at $5.6 \mathrm{GHz}$ as a function of $b / a$, for four drop diameters $(d)$ as indicated; backscatter. The labels $V 90, H 90$, and 0 refer to different linear polarizations of incident radiation, as in Figure 2. Points $X$ represent the $P P$ drops. The label $0, H$ indicates validity for all values of $\alpha$ with $H$ polarization.

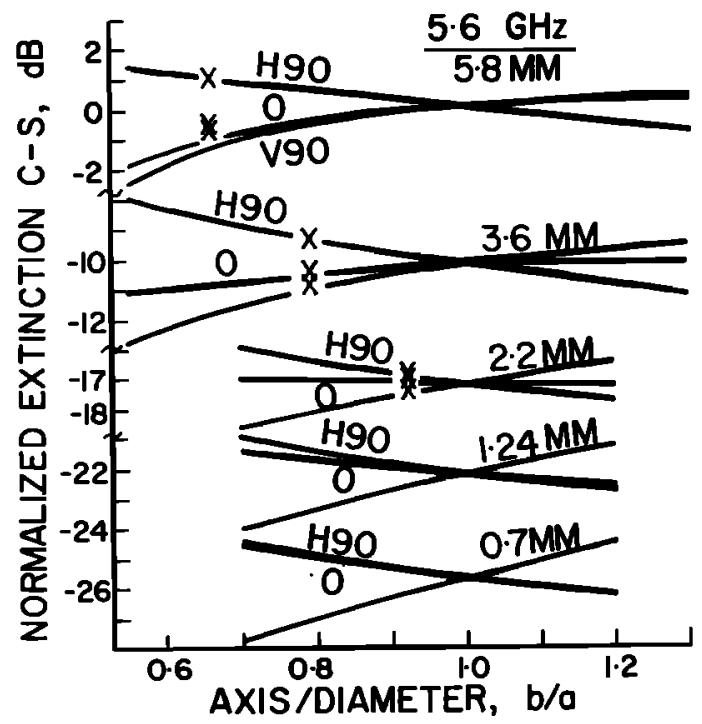

Fig. 6. See caption for Figure 5; extinction. 
were found to remain quite small.

The drop shapes of Pruppacher and Pitter (PP) are nearly oblate spheroids, but are flattened on the underside. Their drop of equivolume spherical diameter $5.8 \mathrm{~mm}$ has $b / a \simeq 0.66$. Depolarization ratios for this drop, and for those of slightly smaller sizes, follow curves which are similar to those of oblate spheroids.

For the frequency $5.6 \mathrm{GHz}$, normalized backscattering and extinction cross sections are plotted against axis / diameter ratio in Figures 5 and 6. These graphs contain trios of lines for orientations $V \mathbf{V 0}$, $\mathrm{H} 90$, and $\mathbf{0}$ as above, for each of four equivolume spherical diameters. For angles of incidence $\alpha$ other than $90^{\circ}$ or 0 , cross sections lie between the appropriate line of $\alpha=90^{\circ}$ and that labelled 0 . Results are added for the larger PP drops: the label 0 refers to incidence upwards onto the concave bottom of the drop. With $\alpha=180^{\circ}$, from the opposite direction, results were very similar. The cross sections are smaller for linear polarization along a small dimension of the obstacle than for the opposite case: the lines of $V 90$ lie below those of $H 90$ for oblate

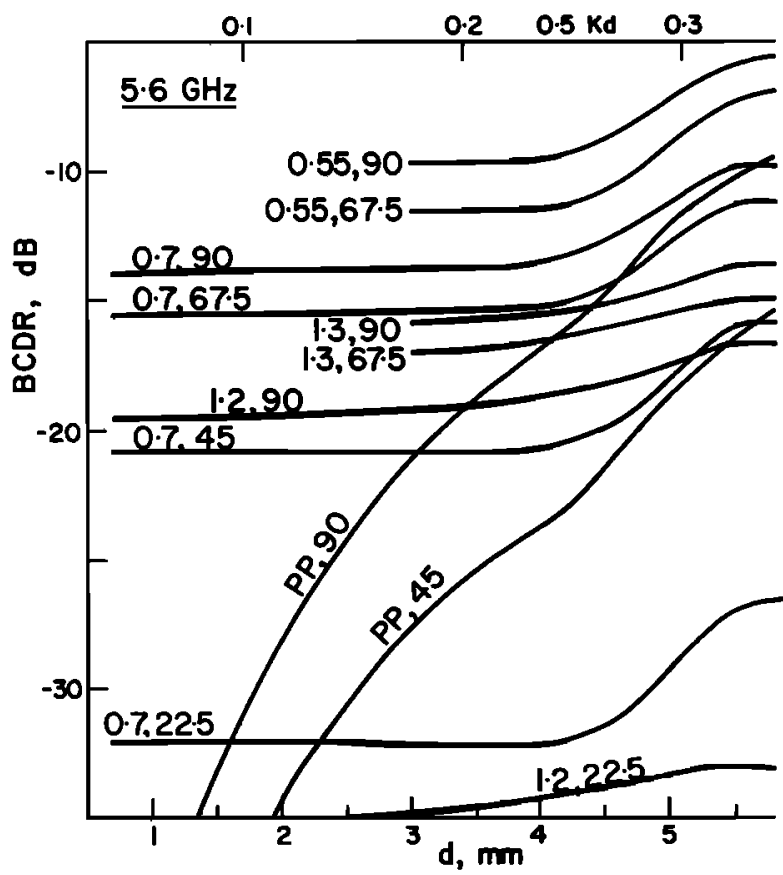

Fig. 7. BCDR as a function of drop diameter (d), at $5.6 \mathrm{GHz}$. Curves are labelled with different axis/diameter ratios and angles of incidence: $b / a, \alpha$ (degrees). PP indicates drops of Pruppacher and Pitter, for $\alpha=90$ and $45^{\circ}$, and $135^{\circ}$ where different from the latter.

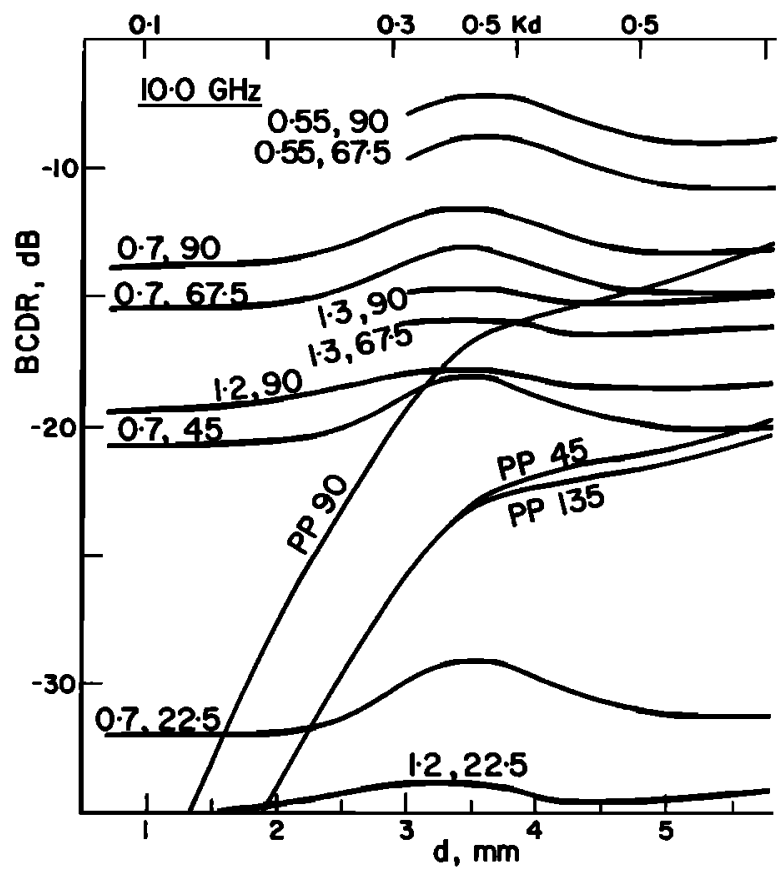

Fig. 8. $B C D R$ as a function of drop diameter, at $10 \mathrm{GHz}$. Curve labels as in Figure 7.

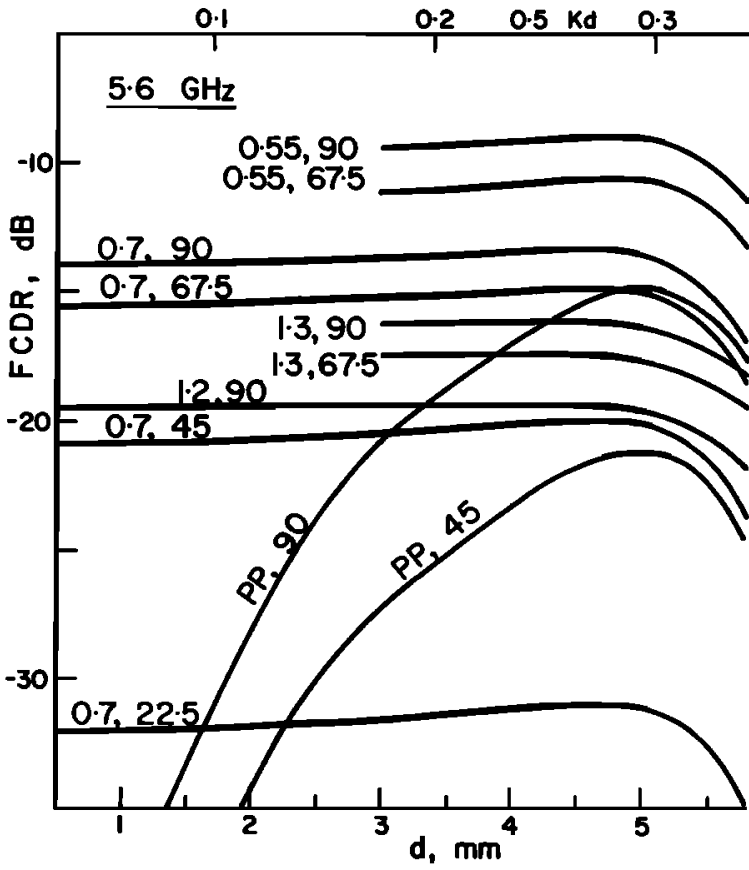

Fig. 9. FCDR as a function of drop diameter, at $5.6 \mathrm{GHz}$. Curves labeled as in Figure 7. 


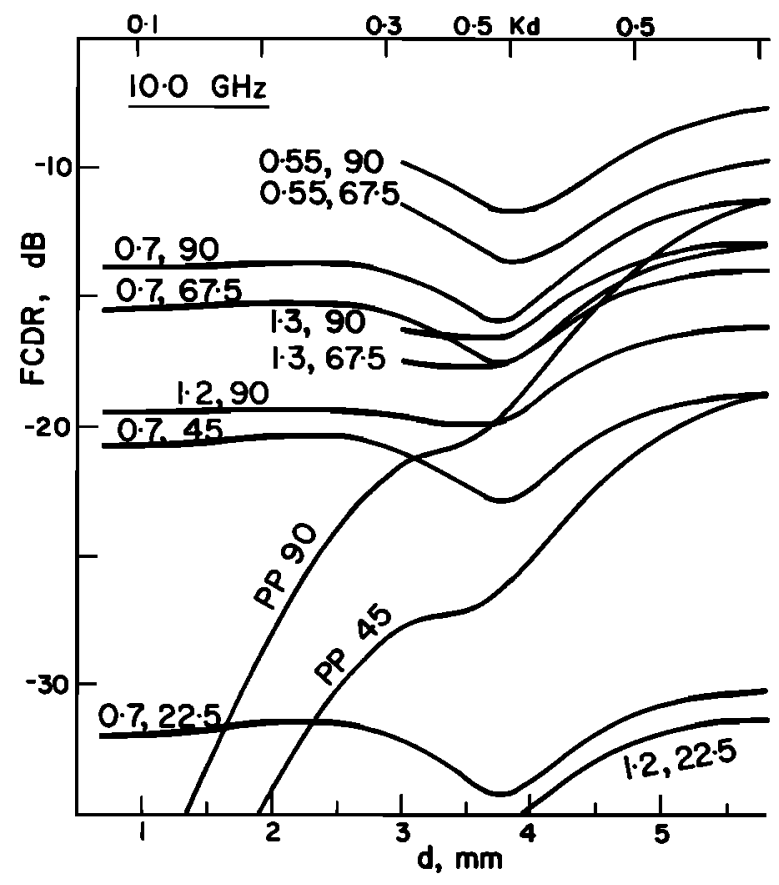

Fig. 10. FCDR as a function of drop diameter, at $10 \mathrm{GHz}$. Curves labeled as in Figure 7.

drops, and above for prolates. Curves for frequencies other than $5.6 \mathrm{GHz}$ are similar to those shown, but different in details.

For the frequencies 5.6 and $10 \mathrm{GHz}$, circular depolarization ratios $B C D R$ and FCDR are shown in Figures 7 through 10. Different curves apply to different shapes and angles of incidence $\alpha$, as indicated, with emphasis on the extremes of shape, and values of $\alpha$ near $90^{\circ}$. Curves are added for the PP drops. Depolarization ratios of practical interest exceed $-10 \mathrm{~dB}$ only for the most distorted drops, and only with $\alpha$ close to $90^{\circ}$. When compared from one frequency to another, they show regular variations according to optical size. The variations with size shown in Figures 7 and 9 are matched quite closely by variations from drop to drop in Figures 5 and 6 , and similar correspondence has been found at other frequencies. With the PP shapes one sees how the smaller asphericities at smaller sizes lead to smaller effects on cross sections and to small depolarizations, as expected.

\section{CONCLUSIONS}

The theory of Waterman $[1965,1969]$ is easy to apply to calculating scattering of microwaves by raindrops. Results compare favorably with observations by Atlas and Wexler [1963].

The scattering characteristics of spheroids appear similar in nature to those of spheres, in the regimes under consideration. For scattering of microwaves by raindrops, marked singularities in the characteristics of depolarization appear at optical size 1.5. The drop shapes of Pruppacher and Pitter [1971] have cross sections and depolarization ratios not very different from those of oblate spheroids, implying that modelling rain in terms of spheroids would be satisfactory.

Radiation of vertical linear polarization commonly is attenuated less than radiation of horizontal polarization. One infers a preference for oblate raindrops and values of $\alpha$ near $90^{\circ}$. As seen from Figures 5 and 6, effects of asphericity on backscattering are greater than on extinction. Thus when using a weather radar to measure heavy rainfall, with $V$ polarization the effect of asphericity is likely to be a reduction of the signal by roughly a $\mathrm{dB}$ or two, and with $H$ polarization a similar enhancement. Means of exploiting this are explored by Seliga and Bringi [1976].

For the largest oblate raindrops, depolarization ratios for backscattering reach a maximum at $C$ band; for drops of diameter $3.5 \mathrm{~mm}$ the maximum is at $X$-band (Figure 4). The presence or absence of very large raindrops might be recognized by measuring depolarization at different frequencies. With a radar operating at $16.5 \mathrm{GHz}$, Hendry and McCormick [1974] have demonstrated the usefulness of radar for distinguishing between different types of precipitation.

To know better the potentialities, particularly respecting depolarization, it seems important to perform integrations over drop spectra using realistic models of rainfall. For this purpose, approximate equations have been found [Warner, 1975], which allow the effects of variable shape and orientation to be taken into account. The theory could readily be applied to axisymmetric hailstones.

\section{APPENDIX A: DETAILS OF COMPUTING}

For obtaining cross sections of spheroidal water drops to a precision of three significant figures, required numbers $\left(m_{1}, n_{1}\right)$ of azimuth and elevation modes are given in Table 1 . Computing times are for five different angles $\alpha$ of incidence for each drop. To allow for $n_{1}$ up to $15,142 \mathrm{~K} 32$-bit words 
TABLE 1. Computational requirements.

\begin{tabular}{|c|c|c|c|c|c|c|}
\hline \multicolumn{2}{|c|}{ For spheroids } & \multirow[b]{2}{*}{$\begin{array}{l}\text { Optical } \\
\text { perimeter } \\
\text { ( } \pi d / \\
\text { wavelength) }\end{array}$} & \multirow[b]{2}{*}{$\begin{array}{l}\text { Axis/ } \\
\text { diameter } \\
\text { (b/a) }\end{array}$} & \multirow[b]{2}{*}{$\begin{array}{l}\text { No: of } \\
\text { azimuth } \\
\text { modes, } \\
{ }_{1} 1\end{array}$} & \multirow[b]{2}{*}{$\begin{array}{l}\text { No. of } \\
\text { elevation } \\
\text { modes, } \\
n_{1}\end{array}$} & \multirow[b]{2}{*}{$\begin{array}{l}\text { Computing } \\
\text { time (s) } \\
\text { on IBM } \\
370 / 195\end{array}$} \\
\hline $\begin{array}{l}\text { Frequency } \\
\text { (GHz) }\end{array}$ & $\begin{array}{l}\text { Equivolume } \\
\text { spherical } \\
\text { diameter } \\
\text { (d, mn) }\end{array}$ & & & & & \\
\hline 2.9 & 5.8 & 0.18 & 0.55 & (3, & 6) & 5 \\
\hline 5.6 & $"$ & 0.34 & $"$ & (3, & 7) & 6 \\
\hline 10.0 & $"$ & 0.6 & $"$ & (4, & 7) & 7 \\
\hline 18.7 & $"$ & 1.1 & $"$ & (5, & 10) & 15 \\
\hline 37.0 & $"$ & 2.2 & 0.55 & (7, & 14) & 40 \\
\hline$-\quad "-$ & $"$ & $"$ & 0.7 & $(6$, & 11) & 21 \\
\hline$"$ & $"$ & $"$ & 0.85 & (6, & 7) & 10 \\
\hline$"$ & $"$ & $"$ & 1.0 & $(m=1$, & 6) & 3 \\
\hline$"$ & $"$ & $"$ & 1.2 & $(5$, & 7) & 8 \\
\hline$"$ & $"$ & $"$ & 1.3 & $(5$, & 11) & 18 \\
\hline$"$ & 3.6 & 1.4 & 0.55 & $(5$, & 9) & 13 \\
\hline$"$ & 2.2 & 0.85 & 0.7 & (4, & 5) & 4 \\
\hline$"$ & 1.24 & 0.48 & $"$ & (3, & 5) & 4 \\
\hline$"$ & 0.7 & 0.27 & " & (3, & 4) & 3 \\
\hline
\end{tabular}

\begin{tabular}{|c|c|c|c|c|c|c|}
\hline \multicolumn{6}{|c|}{ Requirements of $\left(m_{1}, n_{1}\right)$ for } & \multirow[b]{2}{*}{37.0} \\
\hline Frequency & $(\mathrm{GHz})$ & 2.9 & 5.6 & 10.0 & 18.7 & \\
\hline Equivolume & 5.8 & $\left(3,15^{+}\right)$ & $\left(3,15^{+}\right)$ & $(4,10 *)$ & $(5,14 *)$ & $\left(7,20^{+}\right)$ \\
\hline diameter & 5.0 & $\left(3,15^{+}\right)$ & $(3,14)$ & $(4,10)$ & $(4,11)$ & $(7,18)$ \\
\hline \multirow[t]{2}{*}{ d (mn) } & 3.6 & $(3,12)$ & $(3,8)$ & $(3,8)$ & $(4,6)$ & $(5,7)$ \\
\hline & 2.2 & $(3,5)$ & $(3,4)$ & $(3,4)$ & $(3,4)$ & $(4,5)$ \\
\hline
\end{tabular}

* for precision of two significant figures

+ for precision of a few percent

of core storage were used for the whole program. For $\alpha=0$ or $180^{\circ}$, along the axis of rotational symmetry, it is necessary to use only $m=1$.

Requirements for treating the drops of Pruppacher and Pitter [1971] are given in the lower half of the table. As indicated by the symbols * and + , a precision of three significant figures was not attained for the most deformed drop. To get the present results, two slightly different programs were used; the two regimes are separated by a line within the table. The results at $37 \mathrm{GHz}$ were obtained with a program as for the spheroids, with $210 \mathrm{~K}$ of storage to get $\left(m_{1}, n_{1}\right)$ up to $(7,20)$. Results for the larger drops show an asymptotic settling as the maximum value of $n$ is increased. At $2.9 \mathrm{GHz}$ with diameter $5.8 \mathrm{~mm}$, this settling is very slow, being discernible after taking $n_{1}$ upwards from 6 . Here with small optical size, individual matrix elements are small, and it was found appropriate to build quadruple rather than double precision into the solution of equations (12). With capacity for $\left(m_{1}, n_{1}\right)$ up to $(7,17)$, this program required $230 \mathrm{~K}$ of storage. It was used also to confirm results at higher frequencies.

Acknowledgments. This work was done while we were with the Department of Electronic and Electrical Engineering at the University of Birmingham, England. We are grateful to E. D. R. Shearman, T. S. M. Maclean, and colleagues for support and advice, to staff of the Department of Physics, and to the 
Science Research Council, for ready provision of computing facilities.

\section{REFERENCES}

Abramowitz, M., and I. A. Stegun (1968), Handbook of Mathematical Functions, p. 887, Dover, New York.

Atlas, D., and R. Wexler (1963), Backscatter by oblate ice spheroids, J. Atmos. Sci., 20, 48-61.

Buckley, F., and A. A. Maryott (1958), Tables of dielectric dispersion data for pure liquids and dilute solutions, Nat. Bur. Stand. Circ. No. 589, 95 pp., US Government Printing Office, Washington, DC.

Chu, T. S. (1974), Rain-induced cross-polarization at centimeter and millimeter wavelengths, Bell Syst. Tech. J., 53, 1557-1579.

Hendry, A. and G. C. McCormick (1974), Polarization properties of precipitation particles related to storm structure, $J$. Rech. Atmos., 8, 189-200.

Hizal, A., and A. Marincic (1970), New rigorous formulation of electomagnetic scattering from perfectly conducting bodies of arbitrary shape, Proc. Inst. Elec. Eng., 117, 1639-1647.

Inada, H., and M. A. Plonus (1970), The diffracted field contribution to the scattering from a large dense dielectric sphere, IEEE Trans. Antennas Propagat., AP-18, 649-660.

Jones, D. M. A. (1959), The shapes of raindrops, J. Meteorol., 16, 504-510.

Morrison, J. A., and M.-J. Cross (1974), Scattering of a plane electromagnetic wave by axisymmetric raindrops, Bell Syst. Tech. J., 53, 955-1019.

Morse, P. M., and H. Feshbach (1953), Methods of Theoretical Physics, Part II, pp. 1874-1875, McGraw-Hill, New York.
Oguchi, T. (1964), Attenuation of electromagnetic wave due to rain with distorted raindrops, 2, J. Radio Res. Labs. (Japan), II, 19-44.

Oguchi, T. (1973), Attenuation and phase rotation of radio waves due to rain: Calculations at 19.3 and $34.8 \mathrm{GHz}$, Radio Sci., 8, 31-38.

Oguchi, T., and Y. Hosoya (1974), Differential attenuation and differential phase shift of radio waves due to rain: Calculations at microwave and millimeter wave regions, J. Rech. Atmos., 8, $121-128$.

Pruppacher, H. R., and R. L. Pitter (1971), A semi-empirical determination of the shapes of cloud and rain drops, J. Atmos. Sci., 28, 86-94.

Ray, P. S. (1972), Broadband complex refractive indices of ice and water, Appl. Opt., 11, 1836-1844.

Shafai, L., S. J. Towaij, and M. A. K. Hamid (1970), Fast generation of spherical Bessel functions with complex arguments, Electron. Lett., 6, 612-613.

Seliga, T. A., and V. N. Bringi (1976), Potential use of radar differential reflectivity measurements at orthogonal polarizations for measuring precipitation, J. Appl. Meteorol., 15, 69-76.

Taur, R. R. (1975), Rain depolarization measurements on a satellite-earth propagation path at $4 \mathrm{GHz}$, IEEE Trans. Antennas Propagat., AP-23, 854-858.

Warner, C. (1975), Effects of shape and orientation of spheroidal raindrops upon microwave scattering, Electron. Lett., 11, 328-330.

Waterman, P. C. (1965), Matrix formulation of electromagnetic scattering, Proc. IEEE, 53, 805-812.

Waterman, P. C. (1969), Scattering by dielectric obstacles, Alta Freq, 38, 348-352. 